www.psye.com

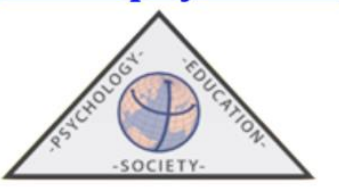

\title{
Marcadores nucleares de la competencia aritmética en preescolares
}

\section{José ORRANTIA ${ }^{1}$, Sara SAN ROMUALDO ${ }^{1}$, Laura MATILLA ${ }^{1}$, Rosario SÁNCHEZ ${ }^{1}$, David. MÚÑEZ ${ }^{2}$; Lieven VERSCHAFFEL ${ }^{3}$}

\author{
${ }^{1}$ Departamento de Psicología Evolutiva y de la Educación, Universidad de Salamanca, España \\ ${ }^{2}$ National Institute of Education. Nanyang, Singapur \\ ${ }^{3}$ Katholieke Universiteit Leuven, Bélgica
}

(Recibido 27 Julio, 2016; Aceptado 10 Noviembre, 2016)

RESUMEN: Las habilidades numéricas y aritméticas son predictores críticos del éxito académico. En trabajos recientes, se ha cuestionado qué habilidades numéricas básicas se relacionan con la ejecución en aritmética, si el procesamiento de magnitudes numéricas no simbólicas o el procesamiento de magnitudes simbólicas. En el presente estudio se tomó una muestra de 104 escolares del segundo curso de Educación Infantil (EI), que completaron una tarea de comparación de magnitudes numéricas no simbólicas, una de comparación de magnitudes numéricas simbólicas y una tarea de enumeración, así como un test estandarizado de rendimiento en matemáticas (TEMA-3). Además, se controlaron habilidades cognitivas generales como inteligencia, velocidad de procesamiento, amplitud de memoria, control inhibitorio y memoria visuo-espacial. Para comprobar si las variables de procesamiento numérico predicen más allá de las variables de control, se realizó un análisis de regresión jerárquica, utilizando como variable dependiente el TEMA-3, e introduciendo las variables de control y las tareas de procesamiento numérico en sucesivos pasos. El modelo explicó el 65.5\% de la varianza. Pero solo la comparación de magnitudes simbólicas y la enumeración contribuyeron a la varianza en ejecución aritmética más allá de las variables de control, mientras que la comparación de magnitudes no simbólicas no contribuyó significativamente. Estos resultados sugieren que un buen conocimiento de los números simbólicos es importante para el desarrollo matemático de los niños, y que particularmente el acceso a la magnitud desde los números simbólicos más que la representación de la magnitud per se es crucial para este desarrollo de la aritmética.

Palabras clave: representación de la magnitud; comparación de magnitudes; enumeración; subitizing; competencia aritmética.

\section{Core markers of arithmetic competence in preschool children}

\begin{abstract}
The numerical and arithmetic skills are critical predictors of academic success. In current studies, it has been questioned what numerical skills relate with arithmetic achievement, whether the non-symbolic numerical magnitudes processing or the symbolic magnitudes processing. In the current study a sample of 104 preschool children was taken. They completed a non-symbolic numerical comparison task, a symbolic numerical comparison task and a dot enumeration task, as well as a standardized arithmetic performance test (TEMA-3). Moreover, general cognitive skills such as
\end{abstract}


intelligence, processing speed, inhibitory control, memory span and visuo-spatial memory, were controlled. To test whether the variables of number processing predict in the absence of the above predictors, it was conducted a hierarchical regression analysis, taking the TEMA-3 as a dependent variable and introducing the other predictors and the numerical processing tasks in next steps. The model explained $65.5 \%$ of the variance. But only the symbolic magnitudes comparison and the enumeration contributed to the arithmetic achievement variance in absence of the control variables, while the non-symbolic magnitudes comparison did not contribute significantly. These results suggest that a good knowledge of symbolic numbers is important to the children's mathematical development, being particularly crucial the access to the magnitude from symbolic numbers more than the magnitude representation per se.

Keywords: magnitude representation; magnitude comparison; enumeration; subitizing; arithmetic achievement.

Correspondencia: José Orrantia, Avda. de la Merced 109-131. 37005. Salamanca orrantia@usal.es.

Numeracy, o como algunos denominan, "literacy cuantitativa" (McCloskey, 2007) incluye las habilidades para razonar con números y otros conceptos aritméticos, y es importante no solo para el éxito académico y profesional de los individuos, sino también para gestionar muchas tareas de la vida cotidiana. Algunas evidencias incluso indican que un rendimiento inferior en estas habilidades se asocia con costos sustanciales para la sociedad en su conjunto (e.g., Butterworth et al., 2011; Duncan et al., 2007). Por lo tanto, entender los mecanismos sobre los que se construyen las habilidades aritméticas se ha convertido recientemente en el foco de atención de numerosas investigaciones. Este planteamiento está inspirado, como sugiere Ansari (2012), en el tremendo progreso llevado a cabo en el campo de la lectura, donde si una competencia básica como el procesamiento fonológico no se desarrolla adecuadamente en la adquisición temprana de la lectura, puede haber un retraso en su aprendizaje, que a su vez puede tener efectos acumulativos en el desarrollo de esta habilidad (véase el llamado "efecto San Mateo" de Stanovich, 1986). Desde este campo de investigación se ha resaltado la importancia de estudiar qué mecanismos sustentan el desarrollo de la lectura para mejorar su adquisición. La cuestión es si en el caso de la aritmética se podría hacer un planteamiento similar, es decir, si se cuenta con evidencias sobre qué competencias básicas podrían constituir la base sobre la que se asienta el desarrollo de las habilidades aritméticas. Aunque esta cuestión no tiene una respuesta definitiva, un número de investigaciones recientes centradas en analizar cómo representamos y procesamos información numérica sugieren que la capacidad para representar y procesar magnitudes podría constituir el sistema fundacional nuclear sobre el que se asientan las habilidades numéricas complejas y la aritmética. El presente artículo se centra en analizar esta cuestión en una muestra de niños de $2^{\circ}$ de EI, ya que en este nivel comienzan a cimentarse las bases para el desarrollo del número y la aritmética. Comenzaremos revisando algunos estudios recientes que han analizado hasta qué punto las diferencias individuales en el procesamiento de magnitudes numéricas se relacionan con diferencias individuales en la ejecución aritmética, para, a parir de los mismos, presentar nuestra aportación a esta cuestión.

\section{Procesamiento de la magnitud numérica y ejecución aritmética}

Aunque los números son una invención del hombre, las habilidades numéricas no son solo el producto de una transmisión cultural. Algunos aspectos de la cognición numérica tienen 
una larga historia en la evolución, hasta el punto de que se plantea que el ser humano (al igual que otras especies animales) está dotado de ciertos mecanismos que le permiten representar y procesar cantidades (magnitudes) numéricas desde que nace, los cuales serían la base sobre la que se asientan las habilidades numéricas transmitidas culturalmente (Barth, La Mont, Lipton\&Spelke, 2005; Dehaene, 2011).

Así, los bebés, los niños, los adultos e incluso los animales son capaces de entender y manipular magnitudes numéricas por medio de representaciones no simbólicas. Y el mecanismo subyacente a estas representaciones es conocido como el sistema numérico aproximado (SNA; Dehaene, 2011), un sistema primitivo de representación no verbal que permite a los individuos procesar magnitudes y cuyo desarrollo no depende de una enseñanza explícita (Feigenson, Dehaene\&Spelke, 2004). La tarea más ampliamente utilizada para analizar la naturaleza del SNA ha sido la comparación de magnitudes, en la que se presenta a los participantes dos conjuntos de puntos para decidir cuál es más numeroso. Los resultados en esta tarea reflejan un efecto de ratio o distancia entre los dos conjuntos de puntos; es decir, es más difícil distinguir un conjunto de 24 puntos de uno de 18 (ratio .75 y distancia 6) que distinguir un conjunto de 24 puntos de uno de 12 (ratio .50 y distancia 12). Estos efectos de ratio o distancia se suelen utilizar como índices de la precisión o agudeza del SNA, aunque el índice más ampliamente utilizado es la fracción de Weber $(w)$, calculada sobre la base de la ejecución de los participantes a lo largo de diferentes ratios; cuanto más pequeño es el tamaño de $w$ más precisa es la representación de la magnitud, lo que también se traduce en menores efectos de ratio y distancia. Y dicho tamaño disminuye con la edad, llevando a representaciones cada vez más precisas (Halberda\&Feigenson, 2008).

También se ha sugerido que el SNA está implicado en el procesamiento de la información numérica simbólica, esto es, cuando los individuos tienen que procesar números simbólicos (eg., dígitos arábigos). Algunos autores han propuesto que cuando se aprenden las representaciones simbólicas exactas de la cantidad, estas se proyectan sobre las representaciones del SNA (e.g., Barth et al., 2009; Lipton\&Spelke, 2005). En otras palabras, cuando se aprenden los símbolos para representar números, estos adquieren significado cuando se asocian con el SNA preexistente (Dehaene, 1992; Mundy\&Gilmore, 2009). De hecho, los efectos de distancia y ratio también se producen en tareas de comparación de magnitudes simbólicas (Moyer\&Landauer, 1967). Y como ocurre con la comparación de magnitudes no simbólicas, estos efectos se reducen con la edad (Holloway\&Ansari, 2009), bien por un incremento en la precisión de las representaciones de la magnitud, o bien por un incremento en la precisión de la proyección entre representaciones simbólicas y no simbólicas. Aunque aún no hay acuerdo entre los investigadores en cuándo y cómo se lleva a cabo esta proyección entre los numerales simbólicos y las representaciones no simbólicas de la numerosidad (ver Leibovich\&Ansari, 2016, para una revisión de esta cuestión), lo cierto es que se considera que el SNA puede jugar un papel importante en el aprendizaje de la aritmética, y que por lo tanto las diferencias individuales en la ejecución del SNA se relaciona con las diferencias en la ejecución aritmética. Un número de trabajos recientes han estudiado esta relación, con resultados mixtos y no concluyentes.

Una de las cuestiones que más debate está generando es qué explica el éxito en la ejecución en aritmética, si la representación de la magnitud per se, esto es, la competencia para procesar magnitudes no simbólicas, o el acceso a la representación de la magnitud desde los 
números simbólicos. Por ejemplo, dos recientes meta-análisis (Chen\& Li, 2014; Fazio, Bailey, Thompson, \&Siegler, 2014) han mostrado una relación moderada, aunque significativa, entre el procesamiento de la magnitud numérica no simbólica y la ejecución en aritmética. Sin embargo, cuando se analizan conjuntamente el procesamiento de magnitudes numéricas no simbólicas con simbólicas, los resultados son menos concluyentes. Por ejemplo, De Smedt, Noël, Gilmore y Ansari (2013) llevaron a cabo una revisión narrativa mostrando que solo el $44 \%$ de los estudios analizados presentaron una relación significativa entre ejecución en aritmética y procesamiento de la magnitud numérica no simbólica, mientras que el $76 \%$ de los estudios relevaron una asociación significativa con el procesamiento de la magnitud numérica simbólica. De la misma manera, otro reciente meta-análisis de Schneider, Beeres et al. (2016) que incluyó tanto medidas de procesamiento de magnitudes numéricas no simbólicas como simbólicas reveló un tamaño del efecto significativamente más grande de la asociación entre ejecución aritmética y medidas que incluyen estímulos simbólicos que aquellas que incluyen estímulos no simbólicos.

Otros autores, sin embargo, argumentan que la habilidad para comparar magnitudes no es la única competencia numérica nuclear sobre la que se asientan las habilidades aritméticas. También se ha sugerido que la habilidad para enumerar eficientemente pequeños conjuntos puede ser una competencia nuclear, ya que la enumeración también requiere acceso a la representación de la magnitud numérica (Reeve, Reynolds, Humberstone, \&Butterworth, 2012). La enumeración evalúa la velocidad y exactitud para identificar conjuntos de puntos, generalmente entre 1 y 8 , y tiene como característica que los conjuntos pequeños $(n \leq 4$, ) se enumeran de manera más rápida y exacta que los conjuntos grandes $(n \geq 4)$, lo que ha llevado a identificar dos sistemas para enumerar (e.g., Hyde, 2011), uno para conjuntos pequeños denominado subitizing, y otro para conjuntos grandes denominado conteo. Y aunque el número de investigaciones es menor respecto a la tarea de comparación de magnitudes, algunos trabajos han mostrado una relación entre la enumeración (tanto en el rango de subitizing como en la tarea global) y la ejecución en aritmética (e.g., Bartelet, Vaessen, Blomert\&Ansari, 2014; Gray \&Reeve, 2014; More \&Ashcraft, 2015).

\section{El presente estudio}

El objetivo de este artículo ha sido analizar hasta qué punto las habilidades para comparar magnitudes numéricas, tanto simbólicas como no simbólicas, y para enumerar conjuntos de ítems pueden ser marcadores diagnósticos de las habilidades aritméticas emergentes en niños preescolares. Hasta donde sabemos, ningún trabajo previo ha analizado en un mismo estudio la relación entre estas habilidades y la ejecución aritmética en esta edad en la que los niños están comenzando a adquirir las habilidades numéricas más básicas. Además, analizamos si estas habilidades predicen más allá de lo que predicen variables cognitivas no numéricas tradicionalmente relacionadas con la ejecución aritmética, tales como la inteligencia, la memoria de trabajo verbal y espacial, la velocidad de procesamiento o el control inhibitorio. Aunque algunas de estas variables han sido incluidas en algunos de los estudios previos, ninguno las ha considerado conjuntamente en un mismo estudio. Por último, analizamos si es la eficacia para procesar magnitudes (reflejado en medidas de velocidad y aciertos) o la precisión o agudeza de la representación de la magnitud (reflejado en los efectos de distancia o ratio) lo que se relaciona con la ejecución matemática 


\section{Método}

\section{Participantes}

En el estudio participaron 106 preescolares de dos escuelas de una ciudad española.Dos participantes fueron excluidos por no completar todas las tareas La muestra estaba compuesta por 54 niños y 50 niñas con una media de edad de 56 meses (DT $=4$ meses). Los padres de los participantes dieron su consentimiento por escrito y se siguieron las normas del Comité de Bioética de la Universidad en la que se desarrolló el estudio.

\section{Instrumento}

Comparación de magnitudes numéricas no simbólicas.

En esta tarea se utilizó una versión de Panamath (thepsychophysicalassessment of number-senteacuity; Halberda\&Ly, 2013). Los participantes tenían que elegir el más numeroso de dos conjuntos de puntos (azules y amarillos) presentados simultáneamente a ambos lados de una pantalla de ordenador apretando las teclas $\mathrm{S}$ (más numeroso a la izquierda) o L (más numeroso a la derecha). El rango de numerosidad iba desde 4 puntos a 15 con 4 ratios diferentes $(.50, .66, .75$ y .86). La prueba constó de 56 ensayos más 4 de práctica. Cada ensayo comenzó con un punto de fijación (1000 ms) seguido por los conjuntos de puntos que permanecieron un tiempo limitado en la pantalla $(2000 \mathrm{~ms})$ para evitar conteo. El siguiente ensayo apareció apretando el investigador la barra espaciadora. En la mitad de los ensayos los puntos amarillos fueron más numerosos y en la otra mitad los azules. Para prevenir el uso de estrategias basadas en variables continuas (tamaño de los puntos, área, luminosidad), el radio por defecto de los puntos fue de 60 píxeles y la variabilidad máxima en el tamaño fue de $\pm 35 \%$; además, en la mitad de los ensayos el tamaño de los puntos y el área decrementó con la numerosidad, y en la otra mitad incrementó. Las medidas utilizadas para esta tarea fueron la proporción de errores como medida de eficacia y la fracción de Weber (para proporciones de aciertos por encima de 0.5 ) como medida de representación de la magnitud.

\section{Comparación de magnitudes simbólicas (1-9)}

Los participantes tenían que elegir lo más rápidamente posible el mayor de dos números arábigos (1 a 9) presentados simultáneamente a ambos lados de una pantalla de ordenador apretando las teclas $\mathrm{S}$ (dígito mayor a la izquierda) o $\mathrm{L}$ (dígito mayor a la derecha). Cada ensayo fue precedido de un punto de fijación $(1000 \mathrm{~ms})$ y los números permanecieron en la pantalla hasta la respuesta. La distancia numérica entre estímulos fue de 1 a 5 y se presentan 8 ensayos para cada una de las distancias para un total de 40 ensayos más 3 de práctica. Las medidas utilizadas para esta tarea fueron, como medidas de eficacia, el tiempo de reacción (TR) medio de respuestas correctas, la proporción de aciertos y un índice de eficacia (en adelante IE) dividiendo el TR por la proporción de aciertos. Como efecto específico de la representación de la magnitud se obtuvo un índice de distancia (ID) para cada participante a partir de la pendiente de una regresión sobre los TR y proporción de aciertos utilizando como 
variable predictora la distancia.

\section{Enumeración de puntos}

A los participantes se les presentaba una serie de puntos negros dispuestos aleatoriamente en una pantalla de ordenador sobre un fondo blanco. El tamaño de los puntos fue constante ( $1 \mathrm{~cm}$ de diámetro) y la numerosidad varió a lo largo de los ensayos ( $\mathrm{n}=1$ a 6 ). Los puntos se presentaron en una cuadrícula $(14$ por $10 \mathrm{~cm})$ de manera pseudo-aleatoria, con una distancia mínima entre puntos de $2 \mathrm{~cm}$ y una distancia máxima de $7 \mathrm{~cm}$. La prueba se compuso de 24 ensayos (4 por cada numerosidad) presentados aleatoriamente, más 4 de práctica. Como los participantes de este estudio son niños muy pequeños para utilizar un dispositivo de voz activada que recoja los TR, se utilizó una grabadora de voz durante toda la prueba para registrar el momento exacto en el que el participante emitía la respuesta. De este modo, aparecía un punto de fijación en el centro de la pantalla (1000 ms) acompañado del sonido de una campana que anunciaba la aparición de cada estímulo. El estímulo permanecía en la pantalla hasta que el participante daba una respuesta y en ese momento, el investigador registraba la respuesta en un pulsador, transcurrido $2000 \mathrm{~ms}$ aparecía el siguiente estímulo. Posteriormente, y utilizando el software Audacity, se calculó de manera exacta el tiempo (en ms) transcurrido desde la aparición del estímulo (campana) y el momento en el que el participante emitió la respuesta registrados ambos en la grabadora. Las medidas utilizadas para esta tarea fueron el TR medio, la proporción de aciertos y el IE, tanto para la tarea global como para el rango de subitizing. Y como efectos específicos de la representación de la magnitud se calcularon para cada participante las pendientes de la regresión sobre los TR y la proporción de errores utilizando como variable predictora el número de puntos, tanto para la tarea global como para el rango de subitizing.

\section{Variables de control}

\section{Inteligencia}

Como medida de la inteligencia se utilizó la versión española del test estandarizado de Matrices Progresivas de Raven (Raven, Court\&Raven, 1992). La medida fue el número de ítems resueltos correctamente.

\section{Velocidad de procesamiento}

En esta tarea se presentaron simultáneamente un cuadrado rojo y uno negro a ambos lados de una pantalla de ordenador y los participantes tenían que señalar lo más rápido posible dónde estaba el cuadrado rojo apretando la tecla S si se encontraba en la izquierda y la letra L si se encontraba en la derecha. La tarea está compuesta por 20 ensayos más 4 de práctica. La medida tomada fue el TR medio de los ensayos correctos. 
Amplitud de memoria verbal

Esta tarea incluyó el recuerdo (directo e inverso) de listas de palabras familiares monosilábicas que variaron en longitud de dos a ocho palabras, con cuatro listas para cada longitud presentadas por el experimentador una palabra por segundo. La tarea finalizó cuando el participante cometía tres errores dentro de una amplitud. Como medida se aplicó 0.25 puntos por lista repetida correctamente.

\section{Memoria espacial}

Para evaluar la memoria espacial se utilizó el test estandarizado de Cubos de Corsi en el que el experimentador señala sucesivamente una secuencia de bloques presentados sobre un tablero con nueve bloques que el participante tiene que reproducir en orden directo e inverso. La tarea comenzó con una secuencia de dos bloques hasta nueve, y cada secuencia incluyó dos ensayos. La tarea finalizó cuando el participante fracasaba en los dos ensayos de una secuencia. A cada ensayo correctamente reproducido se le concedió 1 punto.

\section{Control inhibitorio (go/no-go)}

Se diseñó una versión computarizada de la tarea go/no-go de peces-tiburones. Antes de comenzar la tarea los niños fueron instruidos para pulsar la barra espaciadora cuando vieran un pez en la pantalla del ordenador (ensayos go) y de este modo atraparlo en su red de pescadores y no pulsar ningún botón cuando vieran un tiburón (ensayos no-go), ya que los tiburones podrían romper sus redes. Se presentaron 40 ensayos (el $75 \%$ eran con peces y el $25 \%$ con tiburones). En cada ensayo, el estímulo (pez o tiburón) permanecía en la pantalla durante 1500 ms o hasta que el niño pulsaba la barra. En los estímulos con peces, cuando el niño pulsaba la barra aparecía en la pantalla un dibujo de un pez capturado por una red durante $1000 \mathrm{~ms}$ acompañado de un sonido de burbujas. Cuando aparecía un tiburón en la pantalla y el participante pulsaba erróneamente la barra, aparecía el dibujo de un tiburón rompiendo las redes junto al sonido de un zumbido. Cuando el niño no pulsaba la barra no se daba ningún feedback. Hubo un intervalo inter-estímulos de $1000 \mathrm{~ms}$. La proporción de aciertos (ensayos correctos de go) y falsas alarmas (ensayos incorrectos de no-go) fue utilizado para calcular un índice de sensibilidad (d': la diferencia estandarizada entre la proporción de aciertos y falsas alarmas) calculado a partir del procedimiento propuesto por Macmillan y Creelman (2005).

\section{Ejecución aritmética}

Test de Competencia Matemática Básica (TEMA-3)

La ejecución matemática fue evaluada a partir de la versión española del test estandarizado TEMA-3 (Ginsburg\&Baroody, 2003) para niños de 3 a 8 años que evalúa conceptos y habilidades formales e informales aritméticas. 


\section{Procedimiento}

Los participantes fueron evaluados de manera individual en los centros escolares en una sala aislada de ruidos. Las pruebas se aplicaron en el tercer trimestre del curso en cuatro sesiones de treinta minutos junto con otras pruebas no consideradas en este trabajo. Las tareas que se aplicaron por ordenador fueron diseñadas a partir del software SuperLab, y se presentaron en un ordenador portátil de 15 pulgadas con el teclado adaptado para dejar visibles solo las teclas que los participantes tenían que apretar.

\section{Resultados}

Los ensayos en las tareas computarizadas con respuestas por debajo de $200 \mathrm{~ms}$ o que se alejaron más de 3 desviaciones típicas de la media de los participantes fueron eliminados. En primer lugar se llevaron a cabo diferentes análisis de varianza de medidas repetidas (ANOVA) para comprobar si se cumplen los efectos específicos de las tareas de procesamiento de la magnitud, con la ratio, distancia y número de puntos como factores intra-sujetos para las tareas de comparación de magnitudes no simbólicas, simbólicas y enumeración, respectivamente. Hubo un efecto significativo de la ratio sobre la proporción de aciertos en la comparación de magnitudes no simbólicas, $\mathrm{F}(3,309)=83.11, \mathrm{p}<.0001$, con los participantes siendo menos exactos con ratios mayores. También hubo un efecto de distancia en la comparación de magnitudes simbólicas, tanto sobre los TR, $\mathrm{F}(4,412)=4.82$, $\mathrm{p}<.001$, como sobre la proporción de aciertos, $\mathrm{F}(4,412)=17.04, \mathrm{p}<.0001$, siendo los participantes más lentos y propensos a errores con distancias más cortas. Y en la tarea de enumeración hubo un efecto significativo del número de puntos a enumerar, tanto para los TR1, $\mathrm{F}(5,425)=196.01, \mathrm{p}<.0001$, como para la proporción de aciertos, $\mathrm{F}(5,515)=67.04, \mathrm{p}<.0001$, con más errores y lentitud con numerosidades mayores.

Tabla 1:Correlaciones de Pearson entre las medidas de eficacia y la ejecución aritmética

\begin{tabular}{|c|c|c|c|c|c|c|c|c|c|c|c|}
\hline & 1. & 2. & 3. & 4. & 5. & 6. & 7. & 8. & 9. & 10. & 11. \\
\hline 1. TEMA-3 & & $.420^{* * *}$ & $.526 * *$ & $-.303^{* * *}$ & $-.486 * *$ & $.576 * *$ & $-.376^{* *}$ & $-.637 * *$ & $.466 * *$ & $-.491 *$ & $-.524 * *$ \\
\hline 2. Comp. No Simb (P. Ac.) & & & $.418^{* * *}$ & -.057 & $-.244 *$ & $.316^{* *}$ & $-.394 * *$ & $-.491 * *$ & $.215^{*}$ & $-.432 * *$ & $-.444 * *$ \\
\hline 3. Comp. Simb (P. Ac.) & & & & -.181 & $-.678^{* *}$ & $.233^{*}$ & $-.403 * *$ & $-.480 * *$ & .150 & $-.509 * *$ & $-.516^{* *}$ \\
\hline 4. Comp. Simb (TR) & & & & & $.811 * *$ & -.115 & $.310^{* *}$ & $.326 * *$ & -.064 & $.341^{* *}$ & $.340 * *$ \\
\hline 5. Comp. Simb (IE) & & & & & & -.145 & $.430^{* *}$ & $.459 * *$ & -.087 & $.508 * *$ & $.508 * *$ \\
\hline 6. Enumeración (P. Ac.) & & & & & & & .049 & $-.483 * *$ & $.504 * *$ & $-.248^{*}$ & $-.287 * *$ \\
\hline 7. Enumeración (TR) & & & & & & & & $.814 * *$ & . 001 & .790 ** & $.778 * *$ \\
\hline 8. Enumeración (IE) & & & & & & & & & $-.289 * *$ & $.855^{* *}$ & $.868^{* * *}$ \\
\hline 9. Rango Sub. (P. Ac.) & & & & & & & & & & -.103 & -.184 \\
\hline 10. Rango Sub. (TR) & & & & & & & & & & & $.997 * *$ \\
\hline 11. Rango Sub. (IE) & & & & & & & & & & & \\
\hline
\end{tabular}

Nota: $\mathrm{N}=104$, excepto para enumeración TR y enumeración IE donde $\mathrm{n}=86$

Comp., comparación; No Simb, no simbólico; Simb, simbólico; Sub, subitizing; P. Ac., proporción de aciertos; TR, tiempo de reacción; IE, índice de eficacia. ** $\mathrm{p}<.01, * \mathrm{p}<.05$

\footnotetext{
${ }^{1}$ En este análisis fueron eliminados 18 participantes por cometer errores en todos los ensayos de una numerosidad dada, lo cual ocurrió en todos los casos en el rango de conteo.
} 
Para analizar las relaciones entre las distintas tareas experimentales y la ejecución en aritmética llevamos a cabo dos análisis correlacionales diferenciando medidas de eficacia, tales como TR, proporción de aciertos y IE que combina ambas medidas, y medidas que reflejan representación de la magnitud a partir de la fracción de Weber para comparación no simbólica, el ID en la tarea de comparación simbólica y las pendientes de la tarea de enumeración global y del rango de subitizing.

Tabla 2: Correlaciones de Pearson entre medidas de agudeza de representación de la magnitud y ejecución en aritmética

$\begin{array}{llllllll}1 . & 2 . & 3 . & 4 . & 5 . & 6 . & 7 . & 8 .\end{array}$

$\begin{array}{lccccccc}\text { 1. TEMA-3 } & -.385^{* *} & -.321^{* *} & -.097 & -.369^{* *} & -.452^{* *} & -.426^{* *} & -.415^{* *} \\ \text { 2. Comp. No Simbólica (FW) } & & .307^{* *} & .044 & .178 & .003 & .332^{* *} & .295^{* *} \\ \text { 3. Comp. Simbólica ( } \beta \text { TR) } & & .035 & .093 & .091 & .178 & .334^{* *} \\ \text { 4. Comp. Simbólica ( } \beta \text { ERR) } & & & .081 & .060 & -.005 & .152 \\ \text { 5. Enumeración ( } \beta \text { TR) } & & & & & .097 & .270^{*} & .331^{* *} \\ \text { 6. Enumeración ( } \beta \text { ERR) } & & & & & & .024 & .358^{* *} \\ \text { 7. Rango Subitizing ( } \beta \text { TR) } & & & & & & .073 \\ \text { 8. Rango Subitizing ( } \beta \text { ERR) } & & & & & & \end{array}$

Nota: $\mathrm{N}=104$, excepto para Rango conteo ( $\beta$ TR) donde $\mathrm{n}=86$

$\mathrm{FW}$, fracción de Weber; $\beta$ TR, betas con tiempo de reacción; $\beta$ ERR, betas a partir de errores. ** $\mathrm{p}<.01,{ }^{*} \mathrm{p}<.05$

El análisis de correlación de Pearson entre la ejecución aritmética y las distintas medidas de eficacia son presentadas en la Tabla 1. Como se puede apreciar, todas las medidas consideradas en cada tarea correlacionaron significativamente con la ejecución en aritmética. Para analizar si las distintas tareas predicen la ejecución en aritmética más allá de las variables de control no numéricas se llevó a cabo un análisis de regresión múltiple jerárquica. En el Paso 1 se incluyeron las variables de control no numéricas, y en el Paso 2 la proporción de aciertos de las tareas experimentales. El motivo de utilizar la proporción de aciertos fue poder contar con toda la muestra de participantes (recordemos que en la tarea de enumeración las medidas que incluyeron TR eliminaron de los análisis 18 participantes). El modelo completo explicó un $65.5 \%$ de la varianza (ver Tabla 2 ), $\mathrm{F}(9,94)=19.79, \mathrm{p}<.0001$. Pero más importante, las medidas de eficacia procesando magnitudes aportaron un $16.4 \%$ a la varianza, $\mathrm{F}(4,94)=11.18$, $\mathrm{p}<.0001$. Todas las medidas de eficacia, excepto la proporción de aciertos en comparación no simbólica, hicieron una aportación genuina. 
Tabla 3: Análisis de regresión jerárquica con medidas de eficacia como predictoras

\begin{tabular}{clccc}
\multicolumn{1}{c}{ Pasos } & $\beta$ & $R^{2}$ & $\Delta R^{2}$ \\
\hline \multirow{2}{*}{1} & Variables Control & $.213^{* *}$ & .490 & $.490^{* *}$ \\
& Memoria Espacial & .055 & & \\
& Amplitud memoria & $.218^{* *}$ & & \\
& Inteligencia & -.057 & & \\
& Velocidad Procesamiento & .128 & & $.164^{* *}$ \\
& Control Inhibitorio & & \\
\multirow{2}{*}{2} & Comp. No Simb (P. Ac.) & -.006 & .655 & \\
& Comp. Simbólica (P. Ac.) & $.236^{* *}$ & & \\
& Enumeración (P. Ac.) & $.232^{* *}$ & & \\
& Rango Subitizing (P. Ac.) & $.244^{* *}$ & & \\
& & & & \\
\hline
\end{tabular}

Nota: P. Ac., proporción de aciertos; Comp, comparación; No Simb, no simbólico. ** p<.01 $* \mathrm{p}<.05$

Tabla 4: Análisis de regresión jerárquica con medidas de agudeza de representación de la magnitud como predictoras

\begin{tabular}{clccc} 
Pasos & & \multicolumn{1}{c}{$R^{2}$} & $\Delta R^{2}$ \\
\hline \multirow{2}{*}{1} & Variables Control & $.248^{* *}$ & .470 & $.470^{* *}$ \\
& Memoria Espacial & .036 & & \\
& Amplitud memoria & $.338^{* *}$ & & \\
& Inteligencia & -.095 & & \\
& Velocidad Procesamiento & .124 & & \\
& Control Inhibitorio & & $.084^{* *}$ \\
\multirow{2}{*}{2} & Comp. No Simb (FW) & -.030 & .554 & \\
& Comp. Simbólica ( $\beta$ TR) & -.094 & & \\
& Enumeración ( $\beta$ ERR) & $-.179^{*}$ & & \\
& Rango Subitizing ( $\beta$ ERR) & $-.208^{*}$ & & \\
& & & & \\
\end{tabular}

Nota: FW, Fracción de Weber; $\beta$ TR, betas con tiempo de reacción; $\beta$ ERR, betas a partir de errores; Comp, comparación; No Simb, no simbólico. ${ }^{* *} p<.01,{ }^{*} p<.05$

Por lo que se refiere a las medidas que reflejan la representación de la magnitud, el análisis de correlación de Pearson (ver Tabla 3) volvió a presentar una correlación significativa entre todas las medidas y la ejecución en aritmética, excepto el ID en la comparación simbólica sobre la proporción de aciertos. Y para analizar la contribución a la ejecución en aritmética de las distintas tareas se realizó un análisis de regresión múltiple jerárquica con las variables de control no numéricas en el Paso 1 y como medidas específicas de las tareas experimentales se incluyeron la fracción de Weber, el ID sobre los TR para la comparación simbólica y las pendientes sobre el número de errores para la enumeración global y el rango de subitizing. El modelo completo explicó un 55.4\% de la varianza (ver Tabla 4), $\mathrm{F}(9,94)=12.71$, p $<.0001$, y las medidas de representación de la magnitud aportaron un $8.4 \%$ a la varianza, $\mathrm{F}(4,94)=4.33$, $\mathrm{p}<.01$. En este caso, solo las medidas relacionadas con la enumeración (tarea global y rango de subitizing) hicieron una aportación genuina. 


\section{Discusión}

El objetivo de este estudio fue analizar hasta qué punto medidas relacionadas con las habilidades para procesar y representar magnitudes numéricas tienen una asociación con la ejecución en aritmética en niños preescolares que no han comenzado con la enseñanza formal de la aritmética. Para ello, se utilizaron tres tareas que reflejan estas habilidades: comparación de magnitudes no simbólicas, comparación de magnitudes simbólicas y enumeración, de las cuales se tomaron medidas de eficacia para procesar magnitudes y medidas de agudeza de la representación de la magnitud.

Una cuestión ampliamente debatida es si lo que se asocia con la ejecución en aritmética es la representación de la magnitud per se, o el acceso a la misma desde los números simbólicos. Cuando se consideran medidas de eficacia, los resultados mostraron que todas las medidas correlacionaron significativamente con la ejecución en aritmética. Sin embargo, cuando se controlaron las variables cognitivas no numéricas, la eficacia para comparar magnitudes no simbólicas no contribuyó individualmente a la varianza de la ejecución aritmética más allá de las variables de control y la eficacia para comparar magnitudes simbólicas y enumerar. Esto está en línea con aquellos que sugieren que cuando se combinan medidas de procesamiento de magnitudes numéricas no simbólicas y simbólicas, la asociación con la ejecución aritmética es más robusta para las simbólicas (De Smedt et al., 2013; Schneider et al., 2015). No obstante, el presente estudio añade a los estudios previos que esta asociación se produce incluso cuando se controla un amplio número de variables cognitivas no numéricas. De hecho, la proporción de varianza explicada (65.5\%) es sustancialmente más alta que la encontrada generalmente en los estudios previos, lo cual sugiere que las variables seleccionadas tienen una estrecha relación con la ejecución aritmética. Además, los estudios previos han utilizado principalmente tareas de comparación de magnitudes no simbólicas y simbólicas. En el presente trabajo también se añadió una tarea de enumeración que también requiere acceso a la representación de la magnitud numérica emparejándola a una palabra numérica y que ha sido menos estudiada. Esta tarea también explicó significativamente una parte de la varianza de la ejecución aritmética más allá de las variables de control y de la propia comparación de magnitudes simbólicas, lo que sugiere que ambas tareas reflejan competencias numéricas básicas que contribuyen a la ejecución en aritmética, como proponen Reeve et al. (2012).

Por lo que se refiere a las medidas específicas de las tareas, lo resultados mostraron efectos significativos de ratio, distancia y número de puntos para la comparación de magnitudes numéricas no simbólicas, simbólicas y enumeración respectivamente. Especialmente interesante es el efecto distancia para la comparación de magnitudes numéricas simbólicas, ya que pocos estudios previos lo han analizado en una muestra de niños tan pequeños como los de este estudio. Este efecto distancia sugiere que cuando los niños se encuentran en el inicio del aprendizaje de los números arábigos, estos estos ya comienzan a tener un significado semántico, es decir, los niños ya comienzan a proyectar las representaciones no simbólicas a un nuevo y más preciso sistema para representar magnitudes numéricas.

Los resultados también mostraron que las medidas que reflejan precisión o agudeza de la 
representación de la magnitud, i.e., la fracción de Weber, la pendiente del efecto distancia y la pendiente en la tarea de enumeración (global y rango de subitizing), correlacionaron significativamente con la ejecución aritmética. Sin embargo, cuando se controlaron las variables cognitivas no numéricas, solo los índices de la tarea de enumeración contribuyeron a la varianza de la ejecución en aritmética. El resultado obtenido con la fracción de Weber es consistente con los resultados de las medidas de eficacia para comparar magnitudes numéricas no simbólicas, que no contribuyeron más allá de las demás medidas incluidas en el modelo. La no contribución del efecto de distancia en la comparación de magnitudes simbólicas puede ser debido a la edad de los participantes. Aunque más atrás argumentábamos que el efecto distancia encontrado en los niños de este estudio refleja cierto acceso a la representación de la magnitud, también se ha demostrado que el efecto distancia se reduce con la edad, lo cual puede sugerir que las proyecciones entre representaciones simbólicas y no simbólicas son aún imprecisas a esta edad. Los resultados obtenidos en la tarea de enumeración complementan los obtenidos en las medidas de eficacia y refuerzan la importancia de esta tarea en el aprendizaje temprano de la aritmética. Aunque la aportación de esta tarea a la ejecución matemática es moderada, esta es significativa, aspecto este que se refuerza si tenemos en cuenta el amplio número de tareas de control utilizadas en el presente estudio. Especialmente interesante es la aportación de la medida del rango de subitizing, dado que esta habilidad surge tempranamente en el desarrollo. De hecho, se ha demostrado que los bebés ya son capaces de "subitizar" hasta conjuntos de tres elementos (Feigenson\& Carey, 2003). Además, el subitizing puede constituir la base para la adquisición de conceptos como la cardinalidad (LeCorre\& Carey, 2007), e incluso permite hacer inferencias sobre la numerosidad de los efectos de añadir y quitar conjuntos pequeños hasta tres elementos (Butterworth, 2010).

En definitiva, los resultados del presente estudio sugieren que competencias numéricas básicas como la comparación de magnitudes numéricas (especialmente las simbólicas) y la enumeración pueden tener un papel relevante en la adquisición temprana de la aritmética, y de esta forma constituir marcadores nucleares de la competencia aritmética y por lo tanto tener un valor diagnóstico desde el punto de vista de la prevención y la detección de niños con riesgo de padecer dificultades específicas en el aprendizaje de la aritmética. De hecho, algunos estudios han demostrado que los discalcúlicos presentan déficits específicos tanto en tareas de comparación de magnitudes numéricas simbólicas (e.g., De Smedt\&Gilmore, 2011), como en tareas de enumeración, especialmente en el rango de subitizing (e.g., Landerl, 2013).

\section{Agradecimientos}

Este trabajo fue realizado como parte del proyecto PSI2015-66802-P del Ministerio de Economía y Competitividad.

\section{Referencias}

Ansari, D. (2012). The foundations of numerical and mathematical abilities: a literature review. Global Partnership for Education (GPE) working paper on series, no. 4. Washington DC: World Bank.

Bartelet, D., Vaessen, A., Blomert, L., \& Ansari, D. (2014). What basic number processing measures in kindergarten explain unique variability in first-grade arithmetic proficiency? 
Journal of Experimental Child Psychology, 117, 12-28.

Barth, H., La Mont, K., Lipton, J., \&Spelke, E. S. (2005).Abstract number and arithmetic in preschool children. Proceedings of the National Academy of Sciences of the United States of America, 102, 14116-14121.

Barth, H., Starr, A., \& Sullivan, J. (2009).Children's mappings of large number words to numerosities. Cognitive Development, 24, 248-264.

Butterworth, B., Varma, S., \&Laurillard, D. (2011). Dyscalculia: from brain to education. Science, 332, 1049-1053.

Butterworth, B. (2010). Foundational numerical capacities and the origins of dyscalculia. Trends in Cognitive Science, 14, 534-541.

Cohen-Kadosh, R., Lammertyn, J. \& Izard, V. (2008). Are numbers special? An overview of chronometric, neuroimaging, developmental and comparative studies of magnitude representation. Progress in Neurobiology, 84, 132-147.

Chen, Q., \& Li, J. (2014). Association between individual differences in nonsymbolic number acuity and math performance: A meta-analysis. Acta Psychologica, 148, 163-172.

De Smedt, B. \& Gilmore, C.K. (2011).Defective number module or impaired access?Numerical magnitude processing in first graders with mathematical difficulties. Journal of Experimental Child Psychology, 108, 278-292.

De Smedt, B., Noël, M. P., Gilmore, C., \& Ansari, D. (2013). How do symbolic and nonsymbolic numerical magnitude processing skills relate to individual differences in children's mathematical skills? A review of evidence from brain and behavior. Trends in Neuroscience and Education, 2(2), 48-55.

Dehaene, S. (1992).Varieties of numerical abilities. Cognition, 44(1-2), 1-42.

Dehaene, S. (2011). The number sense: How the mind creates mathematics. New York: Oxford University Press.

Duncan, G. J., Dowsett, C. J., Claessens, A., Magnuson, K., Huston, A., Klebanov, P., et al. (2007). School readiness and later achievement. Developmental Psychology, 43, 14281446.

Fazio, L. K., Bailey, D. H., Thompson, C. A., \&Siegler, R. S. (2014).Relations of different types of numerical magnitude representations to each other and to mathematics achievement. Journal of Experimental Child Psychology, 123, 53-72.

Feigenson, L. \& Carey, S. (2003) Tracking individuals via object files: evidence from infants' manual search. Developmental Scicience, 5, 568-584.

Feigenson, L., Dehaene, S., \&Spelke, E. (2004). Core systems of number. Trends in Cognitive Sciences, 8(7), 307-314.

Ginsburg, H. P., \&Baroody, A. J. (2003). Test of early math ability. Austin, TX: PRO-ED.

Gray, S. A., \& Reeve, R. A. (2014). Preschoolers' dot enumeration abilities are markers of their arithmetic competence. PLoS One, 9(4), e94428.

Halberda, J., \&Feigenson, L. (2008).Developmental change in the acuity of the" Number Sense": The Approximate Number System in 3-, 4-, 5-, and 6-year-olds and adults. Developmental Psychology, 44(5), 1457.

Halberda, J., \& Ly, R. (2013).PANAmath: The psychophysical assessment of number-sense acuity. Unpublished manuscript, Johns Hopkins University. 
Holloway, I. D., \& Ansari, D. (2009).Mapping numerical magnitudes onto symbols: The numerical distance effect and individual differences in children's mathematics achievement. Journal of Experimental Child Psychology, 103, 17-29.

Landerl, K. (2013). Development of numerical processing in children with typical and dyscalculic arithmetic skills -a longitudinal study. Frontiers in Psychology 4, Article 459

Le Corre, M., \& Carey, S. (2007). One, two, three, four, nothing more: An investigation of the conceptual sources of the verbal counting principles. Cognition, 105, 395-438.

Leibovich, T., \& Ansari, D. (2016). The symbol-grounding problem in numerical cognition: A review of theory, evidence, and outstanding questions. Canadian Journal of Experimental Psychology, 70, 12-23.

Lipton, J. S., \&Spelke, E. S. (2005). Preschool children's mapping of number words to nonsymbolicnumerosities. Child Development, 76(5), 978-988.

Macmillan, N. A., \& Creelman, C. D. (2005). Detection Theory: A User's Guide. Mahwah, NJ: Lawrence Erlbaum Associates.

McCloskey, M. (2007).Quantitative literacy and developmental dyscalculias. In D. B. Berch, M. M. M. Mazzocco (Eds.), Why is math so hard for some children (pp. 415-429). Baltimore, MD: Paul H. Brookes.

Moore, A. M., \& Ashcraft, M. H. (2015). Children's mathematical performance: Five cognitive tasks across five grades. Journal of Experimental Child Psychology, 135, 1-24.

Moyer, R. S., \&Landauer, T. K. (1967). Time required for judgements of numerical inequality. Nature, 215(2), 1519-1520.

Mundy, E., \& Gilmore, C. K. (2009). Children's mapping between symbolic and nonsymbolic representations of number.Journal of Experimental Child Psychology, 103, 490-502.

Raven, J. C., Court, J. H., \& Raven, J. (1992). Standard progressive matrices.Oxford Psychologists Press, Oxford, UK.

Reeve, R., Reynolds, F., Humberstone, J., \& Butterworth, B. (2012). Stability and change in markers of core numerical competencies. Journal of Experimental Psychology: General, 141(4), 649.

Schneider, M., Beeres, K., Coban, L., Merz, S., Susan Schmidt, S., Stricker, J., \& De Smedt, B. (2016). Associations of non-symbolic and symbolic numerical magnitude processing with mathematical competence: a meta-analysis. Developmental science. doi:10.1111/desc. 12372

Stanovich, K. E. (1986). Matthew effects in reading: Some consequences of individual differences in the acquisition of literacy. Reading researchquarterly, 21, 360-407. 